\title{
Analysis between Various Random MIMO Systems Model on the Basis of Channel Capacity
}

\author{
Shree Krishna Acharya \\ Department of Electronics and Computer Engineering, Western Regional Campus, Tribhuvan University, \\ Pokhara-16, Lamachour, Nepal; s.kr.acharya@gmail.com
}

Abstract: Finding a good MIMO system model also major issue in Wireless Communication system. It is facing with so many problem, one of the major problem is finding good system model in terms of capacity and transmitting antenna system. In this paper, we analyze the channel capacity of various MIMO system model with some constant SNR level and outage probability. We establish a novel idea for MIMO system models as consider as $2^{N}$ - MIMO system model and find-out change in channel capacity when different transmitting antennas with constant SNR and outage probability. The channel capacity ratio $C C_{R}$ is presented here on the basis of $2^{N}$ - MIMO channel capacity model. Number of transmitted antenna presented in MIMO system is increases is well-known however paper shows change in capacity in simple form.

Keywords: MIMO system Model; $2^{N}-$ MIMO system model; Outage Probability; Channel Capacity Ratio $\left(\mathrm{CC}_{\mathrm{R}}\right)$

\section{INTRODUCTION:}

Multiple Input Multiple Output (MIMO) system is a one of key features of today's wireless system. The transmission and reception of signal of MIMO system are under the Rayleigh Fading. These system are changing randomly and affected by various phenomena, due to this, channel capacity are degraded which is serious problem of modern wireless system. Modern system are leading to the erogdic (average) channel capacity as this is very useful when fluctuation of signal intensity are fast. Maximum data transmission rate is obtain when error probability becomes arbitrary small and two successive symbols contains the independent samples of signal intensity whereas SNR remains constant over duration of large number of symbols.

When signal transmission is random and fluctuations of these signal also known as quasi-static channels, are slow then maximum data transmission rate under a specified outage probability can be known as outage capacity which is more suitable. The outage channel capacity can be calculated by help of [1]. The presented channel capacity ratio between two successive models is under the outage probability and some constant SNR level. The realization of random fading coefficient may be very small when communication over quasi-static fading channels at a given data rate $\mathrm{R}$. In this case, the block (frame) error probability is bounded away from zero no matter whether the block length of symbol tends to infinity [1].

There are various feature of MIMO system but one of the key feature that the channel capacity increase as a multiple antenna system i.e. as numbers of transmit $\left(\mathrm{N}_{\mathrm{T}}\right)$ \& receive $\left(\mathrm{N}_{\mathrm{R}}\right)$ antennas increases then channel capacity increase. No extra additional capacity the increase factor is $\operatorname{Min}\left(N_{T}, N_{R}\right) \operatorname{Min}\left(\mathrm{N}_{\mathrm{T}}\right.$, $\mathrm{N}_{\mathrm{R}}$ ), can be achieved [2]. This paper is concerned with comparison of channel capacity of various MIMO system configuration. Here we analysis between various types of MIMO configuration as $2 \times 2,4 \times$ $4,8 \times 8,16 \times 16,32 \times 32$ and $64 \times 64$ with different SNR levels under outage probability. We analysis various model in $2 \mathrm{~N}-\mathrm{MIMO}$ system model where $\mathrm{N}$ is ranging from 1 to 6 . Here $2^{N}$ gives total number of antennas used in MIMO system and value of $\mathrm{N}$ be same for both transmitting and receiving side. Here we also calculated the channel capacity ratio which is the ratio between two successive modeled channel capacities. 


\section{RANDOM MIMO CHANNEL CAPACITY:}

Let we have a MIMO system with number of transmitting antenna $N_{T}$, number of receiving antenna $N_{R}$ as shown in figure 1. Both of them are narrowband time-variant channel which can be represented as $\left[N_{R} \times N_{T}\right]$ random matrix $\mathrm{H} \times C^{N_{R} \times N_{T}}$, where $\mathrm{H}$ be a random channel parameters. Let us consider that a transmitted vector $\mathrm{X} \in C^{N_{T} \times 1}$ which is composed of $N_{T}$ independent input symbols $\left\{X_{1}, X_{2}, X_{3}, \ldots X_{N-1}, X_{N}\right\}$.Then received signal $Y \in C^{N_{R} \times 1}$ rewritten in matrix forms as follows ${ }^{[2]}$

$$
\mathbf{y}=\sqrt{\frac{E_{x}}{N_{T}}} H x+Z
$$

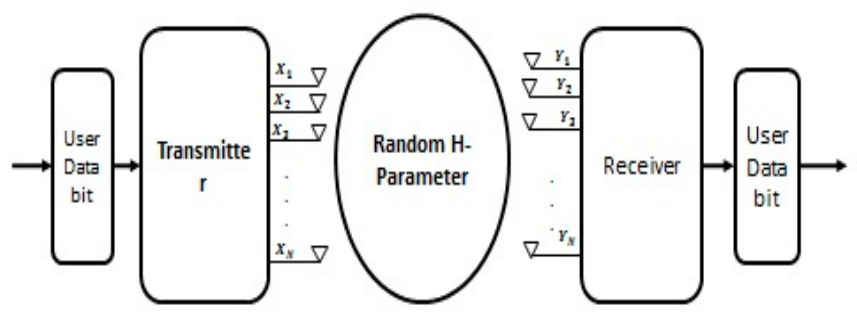

Figure 1 General Block-diagram of MIMO system

Where $\mathrm{Z}=\left\{z_{1}, z_{2}, z_{3}, \ldots z_{N-1}, z_{N}\right\}$ is a noise vector. We assume here this noise vector is a circular symmetric with Zero-Mean Circular Symmetric Complex Gaussian (ZMCSCG) noise signal. The random matrix vector $[\mathrm{H}]$ gives channel capacity which is also randomly timevarying. So that MIMO channel capacity can be given by time average. Here transmitter section doesn't know about Random vector H, spreading the energy equally to all transmitter from one is possible which is an autocorrelation function of transmit signal vector [2]. Due to unknown parameter of random vector $H$ the system doesn't know channel state information

Within the transmission rate $[\mathrm{R}$ bps/Hz], if the decoding error probability cannot be made arbitrary small then the system is called outage. The decoder of $\mathrm{C}(\mathrm{H})$ may commit error only when the channel is in outage. We can say that $\mathrm{C}$-outage channel capacity is the largest possible data rule which yields the equation (2) as less than $\mathrm{C}$. MIMO channel capacity can be has different statistical notation. One of them is outage channel capacity with statistical notation which define on outage probability as [2]

$$
P(C(H) \leq C \in)=\epsilon
$$

From equation (2) we generate the random MIMO channel capacity curves without knowing CSI at transmitter by cumulative distributed function (CDF).

From the mutual Information between transmitter signal and receiver signal we can calculate the MIMO channel capacity. The channel capacity rate is calculated in terms of Eigen vector by using equation (1) as

$$
\text { (C) }=\log _{2}\left(\operatorname{det}\left(I_{N_{R}}+\frac{E_{X}}{N_{T} N_{0}} H H^{H}\right)\right)
$$

\section{Channel Capacity Ratio:}

The channel capacity ratio of this presented $2^{N}$ - MIMO system model can be defined as the ratio of Channel capacity of $2^{N}-$ MIMO system model to the Channel capacity of $2^{N-1}$ MIMO system model with constant SNR and outage probability. 


$$
\mathrm{CCR}=\frac{\text { Channel capacity of } 2^{N}-M I M O}{\text { Channel capacity of } 2^{N-1}-M I M O}
$$

Equation (5) gives channel capacity and $\mathrm{N}$ be the model number of $2^{N}$-MIMO system model which ranging from 1 to $6.2^{N}$ gives total equal number of transmitting and receiving antennas instantaneous capacity [3].

Now the CCR becomes $C C_{R}=\log _{2}\left(\operatorname{det}\left[\frac{I_{N_{R}}+\frac{E_{X}}{N_{T} N_{0}} H H^{H}}{I_{N_{R-1}}+\frac{E_{X}}{N_{T-1} N_{0}} H H^{H}}\right]\right) \ldots \ldots$ (5)

The determinant value of Eigen-matrix is equals to 1 . Hence the equation becomes

$$
C C_{R}=\log _{2}\left(\operatorname{det}\left[\frac{\frac{E_{X}}{N_{T} N_{0}} H H^{H}}{\frac{E_{X}}{N_{T-1} N_{0}} H H^{H}}\right]\right) \ldots
$$

If we consider same receiving signal with noise spectrum and same random vector $H$ then it make independent from matrix and $\log$ equation. And our $C C_{R}$ becomes as function of transmitting antenna.

$C C_{R}=\frac{N_{T}}{N_{T-1}} \ldots(7)$

Where $N_{T}$ is transmitting antenna of $\mathrm{N}_{T} \times \mathrm{N}_{\mathrm{R}}$ and $N_{T-1}$ is transmitting antenna of $N_{T-1} \times$ $N_{R-1}$ antenna system

\section{Simulation Results:}

For comparison between various MIMO systems we might produce the cumulative distribution function (CDF) of the capacity for the random MIMO channel with constant SNR and CSI is not available at the transmitter and receiver because available of CSI also becomes worthless in the channel capacity of MIMO system [2]. The graph between CDF vs. Rate on bps/Hz as shown in figure 2 and 3 where SNR is constant. The SNR value is chosen as $10 \mathrm{dBm}$. The Capacity of random $1 \times 1,2 \times 2,4 \times 4,8 \times 8$ MIMO system model is shown in figure 2 whereas higher capacity of $8 \times 8,16 \times 16,32 \times 32$, and $64 \times 64$ MIMO system is shown in figure 3 . In both of case CSI is not available on the receiver. Due to autocorrelation on transmit signal as increasing in numbers of transmitting and receiving antennas in MIMO system model gives higher data transmission. This system says that better of bandwidth when MIMO system of transmit and receive antennas $\mathrm{N}_{\mathrm{T}} \times \mathrm{N}_{\mathrm{R}}$ then two times uses of $N_{T-1} \times N_{R-1}$ MIMO system when value of $\mathrm{T}$ and $\mathrm{R}$ is equals and power of Two. 

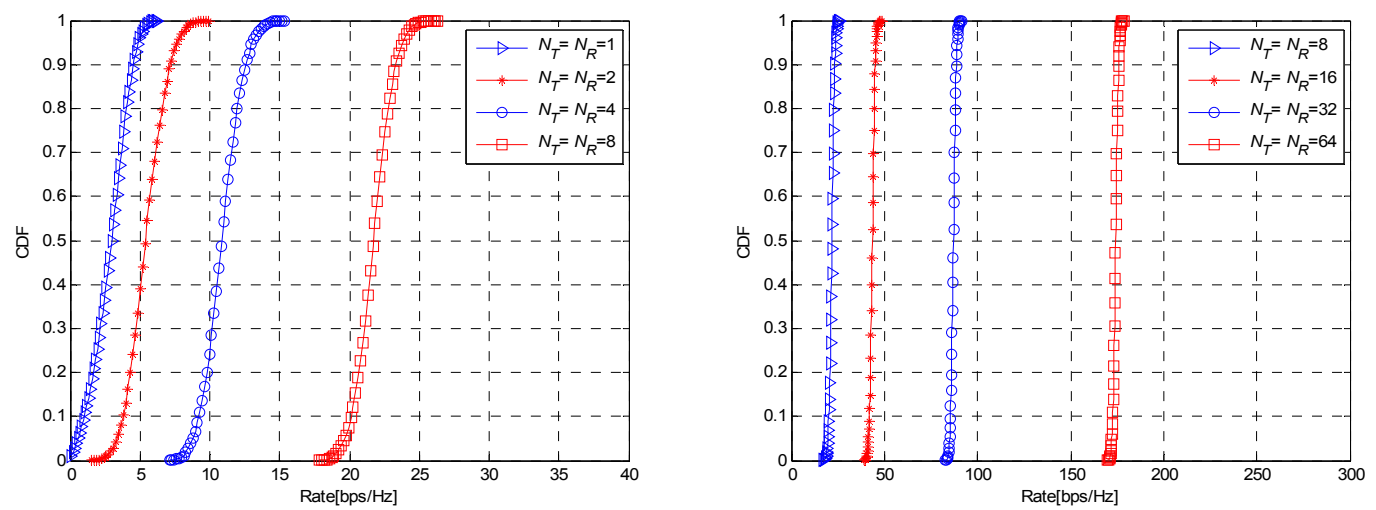

. Figure 2: channel capacity at various transmitting and receinving antennas of MIMO system

\section{Channel Capacity Ratio Table:}

The table shows various MIMO system Model with different SNR level and their respective capacity ratio. Here we compare seven MIMO system model with comparing at three SNR level- $1 \mathrm{~dB}, 5 \mathrm{~dB}$ and $10 \mathrm{~dB}$, and table shows that the channel capacity ratio is greater than two when transmitting antennas are double.. This table is also helpful for analysis of bit data rate in various SNR level. From calculation table we can say that, in terms of channel capacity $N_{T} \times N_{R}$ MIMO system model becomes more beneficial than using two $N_{T} / 2 \times N_{R} / 2$ MIMO system Model, but when value of $N_{T} \times N_{R}$ is higher then use of this is not beneficial then using two $N_{T} / 2 \times N_{R} / 2$ MIMO system model because it gives same performance on channel capacity and higher order system goes more complex. For calculation of channel capacity ratio we use $2^{N}$ model for simplicity. $\mathrm{N}$ is ranging from 1 to 6 and $\mathrm{N}$ gives same amount of Transmitting and receiving antenna. We can say generally $\log _{2}$ configuration of MIMO antennas system. The calculated table shown in Table 1. It shows that with increasing number of transmitter and receiver antennas also improves the performance of data bit rate.

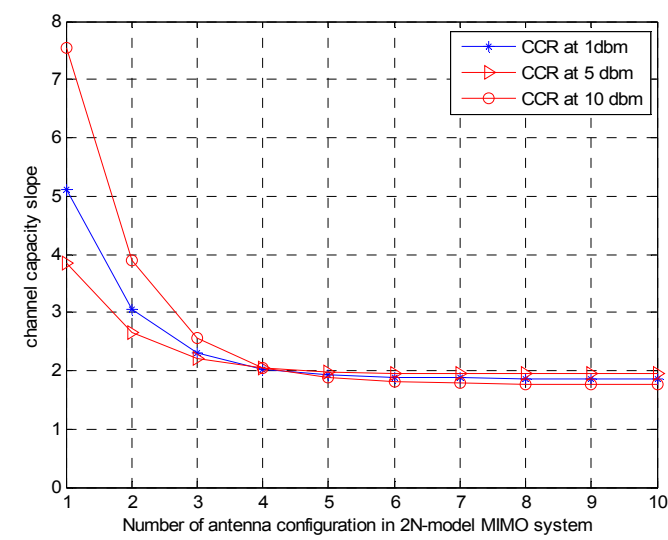


Table 1: Numeric Value of capacity ratio calculation in various MIMO system model

\begin{tabular}{|c|c|c|c|c|c|c|c|}
\hline $\begin{array}{l}\text { S.N } \\
\cdot\end{array}$ & System Model & $\begin{array}{l}\text { Chann } \\
\text { el } \\
\text { Capaci } \\
\text { ty SNR } \\
\text { at } 1 \mathrm{~dB} \\
\end{array}$ & $\begin{array}{l}\text { Channel } \\
\text { Capacit } \\
\text { y SNR } \\
\text { at } 5 \mathrm{~dB}\end{array}$ & $\begin{array}{l}\text { Channel } \\
\text { Capacit } \\
\text { y SNR } \\
\text { at } 10 \mathrm{~dB}\end{array}$ & $\begin{array}{l}\text { Capacit } \\
\text { y ratio } \\
(\mathrm{CCR}) \\
\text { at } \mathrm{SNR} \\
1 \mathrm{~dB}\end{array}$ & $\begin{array}{l}\text { Capacit } \\
\text { y ratio } \\
(\mathrm{CCR}) \\
\text { at } \mathrm{SNR} \\
5 \mathrm{~dB}\end{array}$ & $\begin{array}{l}\text { Capacity } \\
\text { ratio } \\
(C C R) \text { at } \\
\text { SNR } 10 \\
\text { dB }\end{array}$ \\
\hline 1 & $\begin{array}{lll}1 \times 1 & \left(2^{0} \times\right. & \left.2^{0}\right) \mathrm{MIMO} \\
\text { Model } & \end{array}$ & 0.14 & 0.38 & 0.96 & 7.92 & 5.31 & 3.9167 \\
\hline 2 & $\begin{array}{lll}2 \times 2 & \left(2^{1} \times 2^{1}\right) & \text { MIMO } \\
\text { Model } & & \\
\end{array}$ & 1.11 & 2.02 & 3.76 & 2.711 & 2.54 & 2.441 \\
\hline 3 & $4 \times 4\left(2^{2} \times 2^{2}\right)$ MIMO Model & 3.01 & 5.15 & 9.18 & 2.286 & 2.306 & 2.185 \\
\hline 4 & $\begin{array}{l}8 \times 8 \quad\left(2^{3} \times 2^{3}\right) \quad \text { MIMO } \\
\text { Model }\end{array}$ & 6.88 & 11.88 & 20.06 & 2.132 & 2.096 & 2.0867 \\
\hline 5 & $\begin{array}{ll}16 \times 16 & \left(2^{4} \times 2^{4}\right) \mathrm{MIMO} \\
\text { Model } & \\
\end{array}$ & 14.67 & 24.9 & 41.86 & 2.058 & 2.049 & 2.041 \\
\hline 6 & $\begin{array}{ll}32 \times 32 & \left(2^{5} \times 2^{5}\right) \mathrm{MIMO} \\
\text { Model } & \\
\end{array}$ & 30.2 & 51.02 & 85.44 & 2.038 & 2.025 & 2.019 \\
\hline 7 & $\begin{array}{l}64 \times 64\left(2^{6} \times 2^{6}\right) \quad \text { MIMO } \\
\text { Model }\end{array}$ & 61.55 & 103.33 & 172.54 & & & \\
\hline
\end{tabular}

\section{ConCLUSION}

Hence the channel capacity ratio (CCR) is greater than two on each time of calculation it means that it is better to use $2^{N}$ model then using two $2^{N-1}$ model of MIMO system on the sense of channel capacity. But higher values of $\mathrm{N}$, it is not good enough to follow this model. The value of CCR can be useful on practical designing of various MIMO system model design and implementation. With the constant SNR and outage probability as number of transmitter and receiver antennas increases the channel capacity of the MIMO -system model provides better data transmission rate.

\section{7 .REFERENCES}

1) Wei Yang, Giuseppe Durisi, Tobias Koch, Yury Polyanskiy, Quasi-Static MultipleAntennaFading Channels at Finite Blocklength,"

2) Yong Soo Cho $\mid$ Jackwon Kim Won Young Yang | Chung-Gu Kang MIMO-OFDMA Wireless Communication with Matlab,

3) Jelena A. Anastasov Nemanja M. Zdravković Goran T. Djordjevic Outage capacity evaluation of extended generalized-KK fading channel in the presence of random blockage.

4) Ergodic capacity, outage capacity, and information transmission over Rayleigh fading channels Sayantan Choudhury and Jerry D. Gibson Department of Electrical and Computer Engineering.

5) James Osuru Marka Brahim Belhaouari Samirb, Naufal M. Saada Capacity and error probability performance analysis for MIMO MC DS-CDMA system in $\eta-\mu$ fading environment.

6) Muhammad Zia, Tamoor Kiani, Nazar A. Saqib, Tariq Shah, and Hasan Mahmoo Bandwidth-Efficient Selective Retransmission for MIMO-OFDM Systems

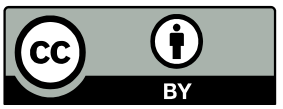

